\title{
Probes to Explore the Individual Perspectives on Technology Use that exist within Sets of Parents
}

\author{
Eleanor Chin Derix \\ Interaction Design and Human Practice Lab \\ University of Technology Sydney \\ NSW, Australia \\ eleanor.derix@googlemail.com
}

\author{
Tuck Wah Leong \\ Interaction Design and Human Practice Lab \\ University of Technology Sydney \\ NSW, Australia \\ tuckwah.leong@uts.edu.au
}

\begin{abstract}
Research reveals that family experiences of technology use in everyday life can be complex and messy, often associated with tension and conflict. This complexity can be intensified when sets of parents have differing individual perspectives on their family's technology use. Exploring these different perspectives, requires an approach that not only considers parents not only as individuals, but also as part of a set. To challenge matters further, parents may not be fully aware of their own attitudes and assumptions relating to technology, let alone of each other's. Parents may also be embarrassed to share details about family conflicts. This methods paper presents a probe study that successfully helped us to explore the individual perspectives on family technology use that exist within sets of parents. It provides an example of an approach to using probes that can reveal the hidden experiences of multiple individuals within a social context. In this way, it contributes an understanding of how we might interrogate the complexities of co-experience.
\end{abstract}

\section{Author Keywords}

Family; parents; technology use; experiences; perspectives; probes.

\section{CCS Concepts}

- Human-centred computing $\rightarrow$ HCI design and evaluation methods

\section{INTRODUCTION}

The pervasive use of digital technologies is increasingly affecting the minutiae of family life [18]. Uncertainties regarding the effects of technology use on child development and family relationships have led to calls for the $\mathrm{HCI}$ community to better understand family experiences of digital technology $[19,44,46]$. One trajectory is to explore the complexities associated with technology use within families e.g.[4, 22, 28]. Research suggests that differences between the experiences, expectations and attitudes of individual family members can contribute to this complexity. Family conflict and tension can arise when parents differ in their approach towards their family's technology use [1, 9, 34]. It is therefore critical that we develop our understanding of these different individual perspectives within sets of parents, and how they are communicated, or negotiated within family life.
However, researching individual perspectives on family experiences presents significant challenges [38]. Firstly, we need to understand the complex social contexts of family relationships in which these experiences take place. In particular, understanding how the needs of individual family members are integrated within the needs of the whole family. Secondly, we need to encourage parents to reflect not only on their own experiences, but also on each other's. Parents may not be fully aware of their own experiences, let alone each other's. This may hold incorrect assumptions about each other's perspectives on family technology use. They might also find it hard to reflect on apparently routine experiences of habitual technology use that occur within the busyness of family life. Furthermore, they could find it embarrassing or uncomfortable to discuss certain experiences, such as those associated with family conflict, or dissatisfaction with aspects of being a parent [11] arising from technology use.

In this methods paper, we present a novel approach to using probes to explore the individual perspectives that exist within sets of parents. While probes have been shown to effectively support research with families, prior work has tended to take either an individualistic, or a collective approach to using them. In other words, some efforts use probes to focus only on individual perspectives, while others design probes to explore the collective (family's) experiences. Instead, we designed our probes to capture a combination of individual and collective responses from each set of parents, in an attempt to reveal a more nuanced understanding of their experiences. We explain that comparing each set of parents' responses, exposed the differently ways in which they perceive experiences of family technology use.

Our findings show how our probes successfully helped to address some of the challenges posed by this research. Firstly, enabling us to discover family dynamics, roles and relationships. Secondly, allowing us to reveal the individual practices and priorities of each parent. Thirdly, helping to raise parents' awareness of each other's perceptions. Finally, prompting parents to reassess their own perceptions. This approach to using probes helped elicit unexpected realizations and reflections on uncomfortable experiences.

Overall, this paper contributes an example of an effective approach to support explorations of domestic life that look beyond individual experiences of technology use, and 
consider some of the complexities including co-experiences. Specifically, our probes enabled us to more deeply explore individual perspectives of parents, regarding their family's experiences of day-to-day technology use. We hope that the knowledge presented in this paper can add to researchers' understanding of how to develop more productive research tools to support inquiries of domestic HCI.

\section{RELATED WORK}

\section{Understanding the Experiences of Parents}

Technology use continues to be increasingly woven into the fabric of family life as it does in society [30]. Meanwhile, uncertainty surrounds the potentially adverse effects technology use might have, especially on children [27, 37] and family relationships $[6,34]$. This has led to efforts within HCI to develop deeper understandings of how families experience technology use within the messiness of everyday life e.g.[13, 46]. However, exploring these experiences presents significant challenges [11].

Some of the challenges associated with uncovering experiences of family technology use, were first described by early researchers of television [7, 38]. They discussed the difficulties of exploring experiences that take place within the social contexts of personal relationships and private domestic settings. Understanding the social contexts of families are particularly complex, as it requires us to consider people as individuals at the same time as considering them as being part of a family. This is because, while families comprise of diverse individuals with different interests and needs [23], being a member of the family unit inherently involves reciprocity and a sense of shared aspirations.

When it comes to domestic technology use, individuals' different experiences, expectations, and attitudes may need to be balanced with those of other family members $[4,55]$. This builds on Battarbee's [3] concept of the co-experience, in which she reminds us that 'people are both individuals and social beings. This is particularly pertinent when considering parents, who not only need to balance their individual interests and desires, but also negotiate the responsibilities, demands and aspirations associated with parenting [31]. This requires parents to consider shared views, modulate opinions, compromise and so on. In order to do this, parents develop expectations, hopes, assumptions and demands on one another [20].

Understanding the individual perspectives on technology use within families is important. As recent research shows, a failure to balance and negotiate between different, even opposing outlooks of individual family members can lead to family tension and conflict $[4,9,55]$. Tensions between sets of parents in particular can be associated with technology use and the different individual attitudes that each parent has towards it $[14,40]$. A set of parents might have to negotiate contrasting individual approaches to implementing family technology rules, including how they each use technology [1, $9,40]$ as well as parenting of their children's technology use
[22, 49]. In addition, children may also share their views on how parents manage and use technology [20]. So, with technology use occupying an increasing amount of individuals' time within everyday family life, many people come to associate it with complex, challenging experiences [9, 24, 47].

Efforts to explore the dynamics of family technology use, offer valuable glimpses into parents' experiences. Traditionally, these predominantly considered the role of parents in mediating and controlling their children's technology use e.g. [4, 34, 50]. However, as technology use has become more ubiquitous, research has also started to consider parents' own use of technology, such as mobile phones [21, 42]. Studies of 'digital motherhood' [16] explore the ways in which technology use is changing parenting practices [2, 32]. While these tend to focus on the use of specific technologies, such as social network sites [39], they begin to reveal the finely balanced role that technology often plays in the lives of parents. For example, the same technologies that parents turn to when seeking or sharing information about their children, offer connection to nonparenting activities and interests [16]. This can help people avoid the isolation often associated with parenting, but can also distract them from looking after their children [21].

These efforts begin to construct an understanding of parents' increasingly complex realities of technology use in family life. However researchers tend to take an individualistic approach to explore the experiences of parents when in fact, their attitudes and approaches to family technology use vary greatly, and are shown to be highly influenced by their relationships and social context. For example, the opinions of family members and friends can affect the types of technology rules set by parents $[14,20,40]$ as well as what they decide is appropriate to share about their children online [1]. The expectations of wider society affects parents' attitudes towards technology use in public, as demonstrated by studies of mobile phone use in family restaurants [45] and of texting at children's playgrounds [21].

While researchers have highlighted how the views of others may affect family's technology use, what is particularly lacking is an understanding of if, and how, sets of parents communicate, negotiate and collaborate on their approach towards their family's technology use [9]. This need for deeper understanding of the experiences of parents correlates with specific calls for a more holistic view of parents' evolving experiences of technology use $[13,25]$ and, more broadly, for HCI research to consider the social elements of experience more thoroughly [3].

\section{Probing Experiences of Family Technology Use}

New tools are required to support research into coexperiences of family technology use, given the significant challenges it presents. In particular, [34] discuss the risk of parents wanting to provide socially desirable responses rather than disclosing family experiences that they might feel uncomfortable or embarrassed about. Furthermore, they 
highlight the critical need to consider the different expectations of individual family members, as well as potential power differentials between them. In addition to these fundamental challenges, others note that intimate contexts require an awareness of privacy concerns [13]. Finally, it has previously proved challenging to integrate research into the busy day-to-day lives of families e.g. due to work commitments of parents [54]. It is perhaps challenges such as these that have encouraged a number of $\mathrm{HCI}$ researchers to turn to probes in order to support their inquiries of family technology use [11].

Probes are playful and open-ended tools [15] used to access aspects of participants' lives by allowing participants to express themselves through collected information [33]. This is often used to supports and stimulate discussions between researchers and participants during contextual interviews. This dialogical approach has been demonstrated effectively within families, promoting the articulation of experiences and behaviours that are usually taken for granted and go unnoticed by participants [23]. The ambiguity of responses can also offer participants privacy, which has led to the use of probes in sensitive settings or with populations that require sensitivity [5]. Their capacity to surface experiential and emotional aspects of interaction design has also been well demonstrated [29]. In this way, a dialogical approach to probes is well placed to help researchers to address some of the challenges presented by exploring co-experiences of technology use within families.

In researching family technology use, one approach has been to design probes to be completed by, and discussed with, an individual family member e.g.[17, 41]. However, [25] advise against taking an individualistic approach when researching families, as it risks promoting Turkle's [48] notion of 'being alone together'. Instead, they suggest taking an approach that considers the needs of the family as a whole. Similar suggestions have been made for more holistic approaches to developing more complete accounts of family experiences with technology [13, 23, 25]. Another approach to researching families has considered the whole family unit. This collective approach involves designing probes as collective family tasks, to be completed by the whole family, in preparation for a collective family interview e.g.[8, 52, 53]. However, seeking a collective response from families assumes that families are homogeneous and overlooks the differences between the individual perspectives of family members [23].

When exploring communication in families, we find that [23] describes an attempt to balance these two approaches by designing one probe to capture the collective perspective of the whole family and another to capture the individual perspective of one family member. Allowing multiple family members to complete the individual probe is recommended, in order to produce a more complex and complete view. We found another example in which probes seem to have been used in a way that combines individual tasks and collective tasks e.g. [51]. However, this approach is not explicitly described, nor is it taken in order to understand how families are currently experiencing their everyday technology use. Rather it is taken in order to supports the design of technologies that mediating intimacy between couples.

As more technologies are brought into homes and the pervasive use of technologies within families is increasingly scrutinised, it becomes critical to adapt our methods to develop a more complex and complete view on these experiences. That is one of the motivators behind our design of a probe study to explore the individual perspectives of family members, in this case, sets of parents.

\section{THE NEED TO EXPLORE PARENTS' PERSPECTIVES}

As we have discussed elsewhere [9], as a precursor to this work we previously held a workshop with parents, to explore their experiences of technology use within family life. This revealed how parents' differing approaches to technology use can result in negative experiences and family conflict. Exploring this further addresses wider calls for better understandings of the interplay between technology use and the complex family dynamics between parents [20, 21, 36]. To the best of our knowledge, there are no explicit examples of methods that explore individual perspectives on family technology use that might exist within sets of parents.

In order to start understanding the social contexts in which parents experience family technology use, we need to take an approach that not only considers them as individuals, but also to part of a set of parents. Our method must also be capable of encouraging parents to reflect on experiences that might seem unremarkable within the habitual technology use of everyday family life. Therefore, we anticipate the significant challenge of encouraging sets of parents to reflect on their own experiences of technology use, and also on each other's.

\section{METHOD - CREATING OPPORTUNITIES TO COMPARE}

We will now describe how we designed our probe study to create opportunities to compare sets of parents' individual perspectives on their family's technology use. Specifically, we will discuss the design of our probes and decisions behind their deployment.

\section{Probe Design - Individual and Collective Responses}

In the absence of explicit examples of how to use probes to explore individual perspectives of multiple family members, we referred to broad guidance on effective probe design (see [10]). However, this guidance tends to be informed by examples in which researchers either take an individualistic or a collective approach to probes. Therefore, probes are either designed to capture individual responses from single participants, or collective responses from multiple participants. When considering how to adapt the use of probes to explore the individual perspectives within sets of 
parents, we attempted to take a balanced approach. This meant designing our probes to capture a variety of individual and collective responses from each set of parents. This built upon suggestions that probe collections work well when they offer participants varying opportunities to respond [33, 53]. We now describe how this approach informed the design of our three probes (i) Family Experience Jar, (ii) Digital Family Tree, (iii) Device Journal (Fig. 1).

\section{Probe 1: Family Experience Jar}

This probe is designed to encourage sets of parents to log their individual experiences of technology use within family life, throughout the study. Each set of parents receives a Jar (Fig. 1, top), along with three small paper notepads which are coloured to denote the type of experiences being logged. Pink for logging positive experiences, blue for negative experiences and yellow for experiences perceived to have both positive and negative aspects. We asked each parent to submit at least one note per day for the duration of the study, inviting them to make additional contributions as-and-when such experiences occurred.

The Jar is designed in such a way so as to prevent the details of the notes inside being read: notes are inserted through a small slit cut into the lid of the Jar, meaning that they must be folded in order to fit. The lid is also glued onto the Jar meaning that notes cannot be removed once they are inserted. Whilst the details of the notes cannot be read, by choosing Jars made of clear glass, participants are able to see contributions amassing over time. The visible colour of the notes inside the Jar provides 'at-a-glance' idea of the types of experiences that had been logged. We hoped this might generate curiosity between parents as to what the other has contributed; encouraging reflection and further participation. Finally, we asked each parent to initial and date their notes to assist us in identifying and comparing their logged experiences.

This probe is inspired by Andell et al.'s [33] stress-relaxation bottle and captures individual responses within a collective container. This is intended as a physical analogy of how we considered participants as being part of a set of parents, and also individuals. While completing this probe, participants would be able to compare the amount and 'mood' of each other's individual responses. When reviewing this completed probe we anticipated being able to compare the individual responses of each set of parents.

\section{Probe 2: Family Tree}

This probe is designed to encourage each parent to express how they see themselves in relation to their family members, as well as in relation to the technologies used within everyday family life. Provided with a piece of A3 paper, participants are asked to create a Family Tree diagram (Fig.1, middle) to illustrate the relationships both between their family members and also the technologies used in everyday family life. We hoped this would help surface insights into how each parent perceives these relationships and into aspects of co-experience. Including technologies in these

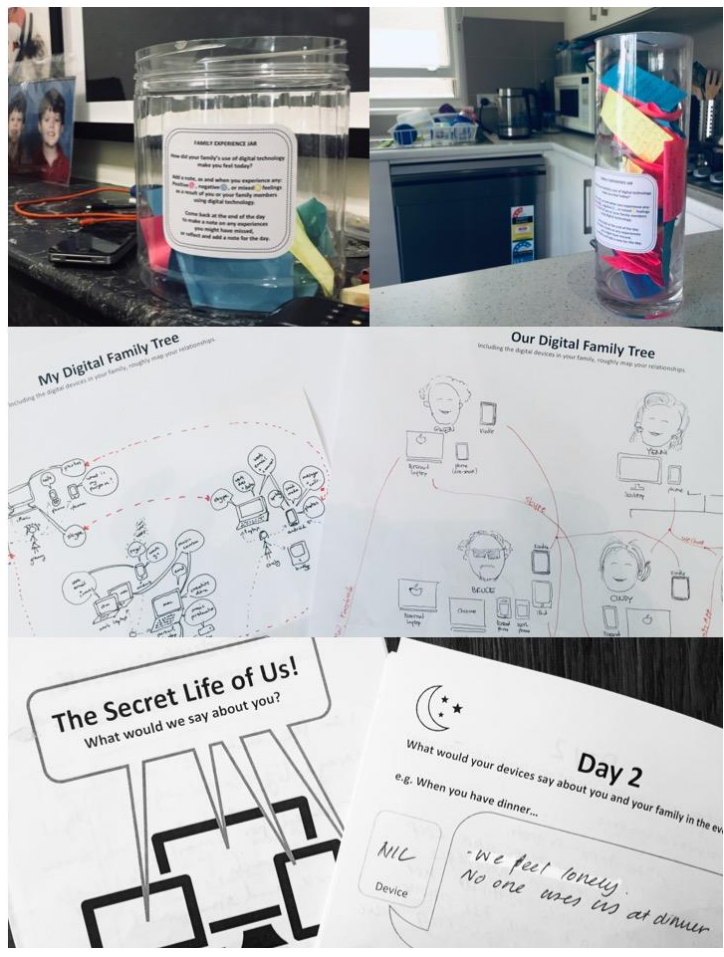

Figure 1. Probe 1 - Family Experience Jar (top), Probe 2 Family Tree (middle), Probe 3 - Device Journal (bottom)

relationships was intended to play into people's tendency to anthropomorphize [12] and assist them to think differently about their family's (often routine, mundane or habitual) technology use.

During the first week of the study, each parent is asked to complete a Family Tree. During the second week, sets of parents are asked to compare their individual responses with one another. Then they are asked to collaborate with each other to complete a shared Family Tree. We asked participants to make a note of any shared outlooks, differences in opinion or even points of contention that might emerge during this process.

This is the probe that most explicitly considers participants as being part of a set of parents, and also individuals. It is designed to capture individual responses from each parent, and then a collective response from each set of parents. To complete this probe, participants would need to compare their individual responses and collaborating on a collective response. These steps are intended to highlight the way in which individual perspectives of parents are communicated and negotiated upon within family life. When reviewing this completed probe we anticipated being able to compare each set of parents' individual responses with each other, and with their collective response.

Probe 3: Device Journal

This probe is designed to encourage parents reconsider their usual perspective on family life. Inspired, in-part, by artifact ecology [26], we devised a comic-style Journal (Fig.1) that 
introduced a fictional context [53] by asking each parent to imagine how their devices experience family life. We hoped this playful probe would enable parents to take a different viewpoint with a refreshed perspective of their family unit and their families' experiences. We hoped that by comparing each set of parents' individually completed journals, deeper insights of habitual technology use would surface that might have otherwise been taken-for-granted, unremarkable, uncomfortable or even socially undesirable.

This probe captures individual responses from each parent within their own Journal. Participants could pick any two days on which to complete this probe and sets of parents were not asked to align, or discuss this task with each other. When reviewing this completed probe we anticipated being able to compare the individual responses of each set of parents.

Probe Deployment - Individual and collective interviews When planning how to deploy our probes, we sought to create a balance between offering opportunities for individual and collective responses, as we had when designing our probe collection. We intentionally held a combination of individual and collective interviews, to consider the individual perspectives within each set of parents.

We decided to hold collective Opening Interviews with each set of parents. We would introduce our probe collection and provide instructions on how and when to complete each probe, which probes required individual or collective responses, and which responses could be discussed or compared. Collective Opening Interviews are particularly appropriate when introducing single, shared probe artefacts such as our Family Experience Jar. In addition, it would

\begin{tabular}{|c|c|c|c|}
\hline Family & Participant & Role & Employment \\
\hline \multirow{2}{*}{ F1 } & P1 & Mother & Full-Time \\
\cline { 2 - 4 } & P2 & Father & Part-Time \\
\hline \multirow{2}{*}{ F2 } & P3 & Mother & Full-Time Parent \\
\cline { 2 - 4 } & P4 & Father & Full-Time \\
\hline \multirow{2}{*}{ F3 } & P5 & Mother & Part-Time \\
\cline { 2 - 4 } & P6 & Father & Full-Time \\
\hline \multirow{2}{*}{ F4 } & P7 & Mother & Part-Time \\
\cline { 2 - 4 } & P8 & Father & Full-Time \\
\hline \multirow{2}{*}{ F5 } & P9 & Mother & Part-Time \\
\cline { 2 - 4 } & P10 & Father & Full-Time \\
\hline \multirow{2}{*}{ F6 } & P11 & Mother & Full-Time Parent \\
\cline { 2 - 4 } & P12 & Mother & Full-Time \\
\hline F7 & P13 & Mother & Part-Time \\
\cline { 2 - 4 } & P14 & Father & Full-Time \\
\hline \multirow{2}{*}{ F8 } & P15 & Mother & Part-Time \\
\cline { 2 - 4 } & P16 & Grandma & Retired \\
\cline { 2 - 4 } & P17 & Aunty & Disability \\
\hline
\end{tabular}

Table 1. Participants allow our participants to identify and introduce themselves as part of a set of parents, and part of a family. This was important given the overall research topic of understanding experiences of family technology use.

We decided to hold individual Closing Interviews with each parent on their own, rather than with sets of parents. This decision was informed by the findings of our preliminary workshop. We hoped it would encourage participants to be more candid and ensure that we were able to explore the different perspectives of each parent. In case a parent might be less candid through fear of us disclosing their opinions during the other parent's Closing Interview, we assured them that their discussions would remain private.

\section{Participants}

This research was conducted in accordance with ethics approval from [University name]. We recruited 17 participants (P1-P17), representing eight families (F1-F8) in which there was at least one child under the age of twelve years (see Table 1). We were cognizant of the broad and diverse range of family compositions [13] and, as is standard in HCI, defined family either as a unit of people living in a home together, or who are related to each other [25]. We acknowledge that many arrangements of parenting exist. For example, F8 consists of a single mother, aunty and grandmother who live together and share responsibility for raising three children.

\section{Study Outline}

The study was conducted over 14 days (see Fig. 2). On Day 1 we conducted semi-structured Opening Interviews with each of the eight set of parents. This took place at their family home and lasted between 60-90 minutes. Each parent introduced themselves and their family, before briefly discussing aspects of technology use within broader family life, including routines, attitudes and expectations. We then introduced our probe collection and explained that they had 10-12 days to complete the probes, before we would collect them.

After collecting completed probes, we reviewed our participants' responses in order to identify interesting questions to be discussed during the semi-structured Closing Interviews held with each of our 17 participants on Day 14. Each Closing Interview lasted between 50-70 minutes and took place, once again, at family homes. This was a researcher-participant co-exploration of the completed probe activities, to make sense and to reflect, retrospectively, on their use of the probes. Also, this interview gave us the opportunity to seek clarifications of certain responses we found interesting when reviewing the completed probes. 


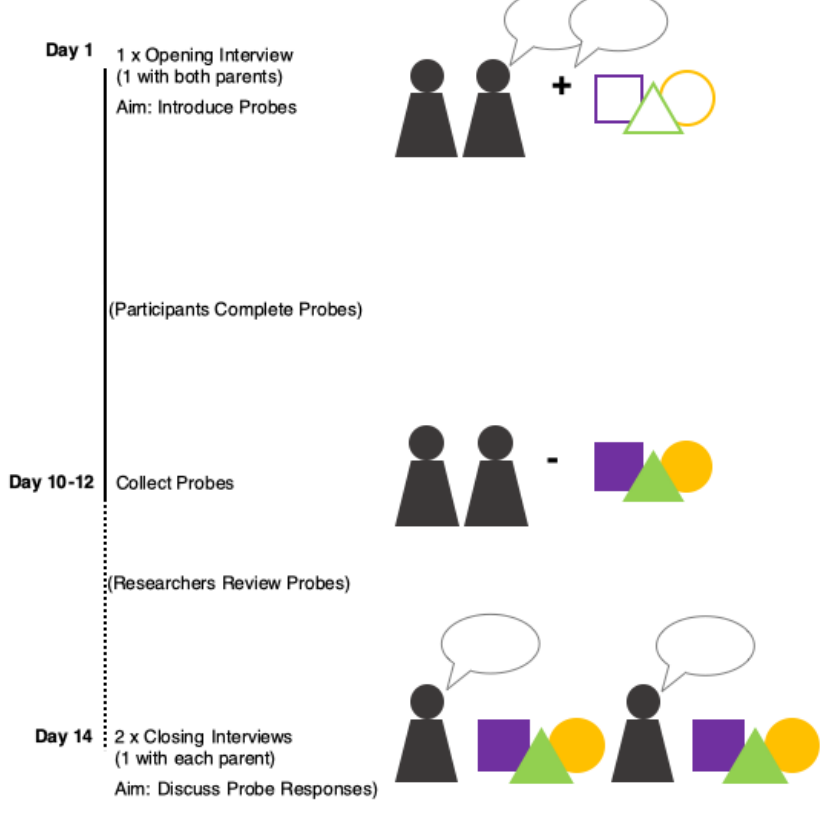

Figure 2. Study Outline

Data Collection and Analysis

After collecting the completed probes, we reviewed them in order to inform Closing Interviews. Firstly, we reviewed the responses of our 17 participants individually. Secondly, we reviewed them as eight set of parents, comparing one parent's responses with the other's. As such, we began to build a picture of, and identify questions relating not only to 17 individual parents, but to eight distinct sets of parents, and to some extent, eight distinct families.

We audio-recorded all interviews and took handwritten notes to support analysis. We used open coding to analyse these data and generated codes to reflect a variety of attitudes and approaches to their family's technology use. These codes combined to create themes that will be reported in future work. For the purpose of this methods paper, we focus on how our approach to using probes helped us to explore the individual perspectives on technology use that exist within sets of parents.

\section{FINDINGS}

In order to highlight the effectiveness of our probe study in enabling us to develop deeper understandings of parents' individual perspectives on their family's technology use, we draw on how participants responded to our probes, as well as on how they reflected upon these responses during Closing Interviews.

As anticipated, when we received and reviewed completed probes, we were able to compare the individual responses of each set of parents. We found that our probes were able to capture the internal dialogues of each parent, by encouraging them to reflect from different, sometimes novel, perspectives. For example, by asking them to imagine how particular technologies perceive family life, our Device
Journal probe prompted them to consider and even reassess their views, revealing usually hidden experiences of family technology use. We were then able to compare these internal dialogues and discuss them during Closing Interviews.

In the case of our Family Tree probe, we were also able to compare each set of parents' individual responses with their collective response. As well as enabling us to compare the individual perspectives that exist within sets of parents, this also allowed us to identify ways in which these different perspectives might be communicated, and negotiated within family life. Participants had been asked to take notice of any interesting conversations, surprising realisations or tensions while completing this probe. This enabled us to ask them about their experience of this process, as we highlighted interesting similarities and differences between their responses during Closing Interviews.

When we interviewed participants, we heard many stories about the differing ways that a set of parents might perceive technology use, and its role within their family. We also surfaced conflicting attitudes about the ways in which technology use might affect their family's relationships. This included elaborate, unexpected realisations that participants sometimes found to be emotional, and even surprising. During these discussions it became clear that our collection of probes had been used successfully to overcome some of the challenges posed by attempting to compare sets of parents' individual perspectives on family technology use. Firstly, discovering family dynamics, roles and relationships. Secondly, revealing parents' individual practices and priorities. Thirdly, raising parents' awareness of each other's perceptions. Finally, prompting parents to reassess their own perceptions.

Discovering Family Dynamics, Roles and Relationships Comparing the individual responses of each set of parents to our probe collection enabled us to garner a sense of the dynamics between each set parents, and their families, insofar as how technology is integrated into their daily practices and routines. Responses to our Family Experience Jars in particular, revealed clues about the role of each parent within their family. We discovered, for example, that one parent tended to log more work-related experiences while the other focused on social, domestic or child-related experiences.

This influence of familial roles was also evident, though perhaps less explicitly, when comparing the degree to which each parent had engaged with the probes. In almost all households, one parent responded more comprehensively than the other. This tended to be the parent who spent more time at home with the children compared to the other parent, who was usually out at work during weekdays. This was visible, for example, in the significant difference between the number of notes each parent contributed to their Family Experience Jar, or by the disparity between the care and detail with which each parent had drawn their Family Tree. 
While we had asked each set of parents to work together to create a collective Family Tree, we found that several collective responses looked very similar to one parent's individual response. We also noticed that some collective responses were missing. During closing interviews, several parents explained that on comparing their individual Family Trees with each other, one parent had conceded that the other parent's diagram was actually more accurate than their own. This individual response had then been either redrawn as a collective response, or used in lieu. In all of these cases, the individual response that was reappropriated as a collective response was created by the parent who held most domestic and child-caring responsibilities. While these explanations might give rise to suspicions of a lack of time or engagement, they may also hint at the true nature of contested opinions, dominant viewpoints and complex negotiations that exist within families, such as one parent's views being more dominant.

By comparing the individual responses of each set of parents we were also able to identify and interrogate instances in which a set of parents describe the same act of technology use. In some instances, we discovered clues about our participants' relationships, or how they perceive their relationships. For example, correlating notes in P7 and P8's Jar, both written on pink paper, describe a shared, intimate experience that both perceive to be positive, "watched Netflix with P7 in bed together" (P8, Jar) and "watched a nice movie on Netflix, me and P8, 2 nights in a row! (-)' (P7, Jar). By contrast, another set of notes expose their conflicting perceptions, with $\mathrm{P} 8$ 's pink note positively describing "binge watching Netflix (alone time)" (P8, Jar) and P7's blue note logging her negative perception of the same experience " $P 8$ spent the whole evening after work watching Netflix" (P7, Jar).

Clues about family dynamics and relationships could also be found when comparing differing individual attitudes of parents towards experiences of technology use involving their children. For example, P11's pink notes describe her positive experiences, "we all watched some kids TV in bed having a cuddle" (P11, Jar) and "while I showered, the boys played games on my phone" (P11, Jar) while P12's blue notes portray these experiences as negative "using $T V$ for calming kids down" (P12, Jar) and "using phone to calm kids" (P12, Jar).

By comparing each set of parents' individual responses, our probes allowed us to more thoroughly explore how each parent perceives their experiences of technology use in family life. This helped to surface deep, candid and interesting reflections by our participants that we could in turn, also compare. For example, during each of their closing Interviews, we asked P7 and P8 to expand on entries they have made in their individual Journals and uncovered contested beliefs: "I can confidently assume that if I became P7's phone for a week I wouldn't be uncovering anything." (P8, Int.) compared to "My phone would know that P8 is spoilt, he's a lucky guy to have a family like us...he would know that from the amount searches I do trying to work him out." (P7, Int.). Disclosures such as these provide insights into family relationships and also highlight the extent to which technology use plays a role within them.

\section{Revealing Parents' Individual Practices and Priorities}

Comparing the individual responses to our probes also helped to reveal the different individual practices and priorities within each set of parents, regarding technology use. For instance, we found Device Journals entries portraying each parent's smartphones as having very different experiences to one another. For example, comparing "I am the centre of P1's life!...I never leave his side or get switched off." (P1, Journal) with, "I am so quiet. $P 2$ almost always mutes me...the grubby little hands (of the kids) that use me sometimes can be rough and have dropped me sometimes." (P2, Journal) reveals the different attitudes and practices of each parent.

Almost all parents describe the television as the device that would know most about their family. Their Journal entries concerning television use also reveal similarities and differences between the individual practices and priorities that exist within sets of parents. For instance, in P5 and P6's Journal entries, we find clues that monitoring their children's technology use is primarily the concern of P5. She imagines their TV to say, "The kids get to watch me while Mum (P5) makes dinner, or in the afternoon on weekends, but not in the mornings...Sometimes Mum streams Cosmic Kids or GoNoodle so that she doesn't feel guilty about kids' screentime." (P5, Journal). In contrast, P6 focuses on the functionality of technology and writes, "I'm the TV, I'm supposed to be part of the smart home setup but all I do is cartoons before dinner." (P6, Journal).

By comparing individual responses to our Family Tree probe, we were able to reveal broader perceptions of technology use within family life. For instance, often, one parent took a people-centric view by drawing connections between faces of family members, while the other took a more technology-centric view by drawing connections between devices.

Raising Parents' Awareness of Each Other's Perceptions The Family Tree probe involved the sharing and discussion of individual responses within each set of parents, before each set could collaborate on a collective response. During Closing Interviews, we found that this process had helped to raise parents' awareness of each other's perceptions on technology use. For instance, in P5's interview, she explained: "When I put together my Family Tree, the relationships are always in terms of the people relationships. The devices facilitate those relationships... whereas P6's is more about the connections between the devices themselves. It was hard to marry them together because of that. They were similar but they had such different focuses. "' (P6, Int.). 
By becoming aware of how the other parent had illustrated their Family Tree, some of our participants had been prompted to re-examine family technology practices that they had previously taken for granted. Several parents talked about how this task had spurred conversations with each other that had led to various new-found realisations about family technology use. For example, P8 explained how collaborating on a collective Family Tree had prompted him to reassess: "I thought that it was a family desktop, but (creating) Our Family Tree made me realise that it's really just me who uses it. I recognise now that these devices are more personal than shared. I realised that everyone in the family has their own (technological) companion" (P8, Int.).

Others discussed how these conversations had exposed conflicting perspectives of technology use. For example, "P10 will tell you a different story...I am surprised at P10's self-opinion of her own use. She doesn't think she uses (her smartphone) that much, but I really do. The (probe) gave me a legitimate lens to have a look at that." (P9). Several participants expressed similar appreciation of the opportunities that this probe created, to discuss perceptions of technology use with the other parent in their family.

\section{Prompting Parents to Reassess Their Own Perceptions}

Asking our participants to collaborate on a collective response to our Family Tree probe demanded a deeper level of comparison by parents of each other's individual efforts. Though challenging, this negotiation of individual perspectives encouraged greater understanding and reflection, not only of one another's perceptions, but also of their own. We found that this facilitated more interesting discussions and surfaced interesting realisations during our Closing Interviews.

It also prompted some parents to reassess assumptions they had made about their families' technology use. For example, P12 described how he was surprised to learn about the central role TV played in his family, realising that his family spent more time watching TV in his absence than he had previously imagined, "I saw that the TV is central to the family, though I don't have any connection to it personally" (P12, Int.). We noticed several participants were similarly surprised to learn that their assumptions about their families' technology use were not always right. For example, P2 who allows her children to access her phone had always assumed that her husband did the same. However, in her Closing Interview, she described her surprise at noticing that her husband's Family Tree showed no connection between his phone and the children. This had prompted her to ask her husband about this and learn for the first time that he did not, in fact, allow their children to use his phone since he considered it to be a work tool. In this way, asking parents to compare their individual responses had created opportunities for conversation between parents and raised awareness of different perspectives on technology use that tend to be overlooked in day-to-day family life.

\section{DISCUSSION}

Our work suggests that using probes in a way that both considers participants as individuals, and well as being part of a family unit, can help to uncover challenging but important aspects of the family dynamics surrounding technology use. This is evident from our participants responses presented in the findings, which illustrate the extent to which our probe study enabled us to compare, explore and unpack the individual perspectives on technology use that exist within sets of parents. As such, this paper provides HCI researchers and interaction designers with a valuable example of how to use probes to productively research the complex experiences of multiple people within family groups.

Our findings describe how our novel way of using probes helped us to address several challenges posed by this research. Firstly, discovering family dynamics, roles and relationships. Secondly, revealing parents' individual practices and priorities. Thirdly, raising parents' awareness of each other's perceptions. Finally, prompting parents to reassess their own perceptions. This enabled us to surface a more complex and complete view of technology use within the lives of our participants and their families. As well as allowing us to comparing the individual perspectives on family technology use that exist within sets of parents, our probes helped us to examine how these perspectives are communicated and negotiated within family life.

Our review of related literature acknowledges an established practice within HCI of using probes in a dialogical approach to support and stimulate discussions between researchers and participants in follow-up interviews [11]. This approach was developed by primarily considering individual experiences of technology, and when working with families, relying solely on responses from individual participants overlooks complex family dynamics and, ultimately, the needs of the whole family [23]. While researchers have sought to correct this by taking a collective approach in which multiple family members complete probes together before discussing responses in group interviews, this neglects the diverse and potentially conflicting perspectives of individual family members [11, 23]. In our efforts we sought a balance between an individualistic and a collective dialogical approach to probes.

This balanced approach considered participants not only as part of a set of parents, but also as individual people. Therefore, as we have described, our use of probes slightly adapted the conventional dialogical approach by designing a probe collection capable of capturing a combination of individual and collective responses. Heeding advice on how to create varied probe collections [53], we designed each of our three probes to capture this combination of responses in different ways, and to varying extents. We had hoped that this would create a range of opportunities to compare the responses of each sets of parents. 


\section{Combining individual and collective probe responses}

While probes that ask people to log their individual experiences are commonly designed as personal diaries [33] our Family Experience Jar probe provided each set of parents with a shared receptacle in which to deposit their individual notes. This physical analogy of the individual perspectives that exist within each set of parents helped to communicate the research topic to participants, thus helping to create an easy entry point for participants to start engaging with our probe collection. By making the notes visible within the Jar, we allowed sets of parents to get a sense of how much one another was engaging with the probe, and the types of experiences that they were logging. Our findings show that this aroused curiosity in our participants and helped to raise participants' awareness of each other's perspectives. We believe that designing probes that capture individual responses within a shared physical object can help to engage multiple people when working with families.

When attempting to compare individual responses from multiple people that are captured in a shared receptacle, it is of course necessary for researchers to be able to identify each participant's individual contributions. We asked participants to initial each of their notes, which allowed us to easily to compare the extent to which each parent had engaged with the activity and the types of experiences that each parent had recorded. This helped in revealing the individual practices and priorities of each parent. By also asking participants to include the date on each of their notes we were able to more precisely compare each set of parents' individual responses, and identify correlating notes describing each parent's version of the same incident. As described in the findings, this allowed us to interrogate differing individual perceptions of a particular co-experience and to discover aspects of family dynamics, relationships and roles. Although occasional examples do exist of probes that capture individual responses from multiple people [33], accounts of their use do not explicitly discuss the use of probes to explore the individual perspectives of multiple family members, or to compare their perceptions of the same experience.

In contrast to our Family Experience Jar probe, each parent recorded their individual responses in their own individual Device Journal. This Journal deviates from conventional diary probes [33] asking participants to record the imagined experiences of devices regularly used by members of their family. Using probes to introduce fictional contexts in this way has been discussed as a means of enabling participants to remove themselves from the constraints of reality, and to express complex ideas [53]. Whilst we have found no explicit accounts of using such probes to explore the individual perspectives within families, our findings indicate that fictional contexts might indeed help encourage family members to consider each other's perspectives. By allowing parents to take a more detached position, this probe also revealed clues about sensitive subjects, such as family conflict. These responses helped us to broach these subjects with participants during Closing Interviews, and elicit revelations about family dynamics, roles and relationships.

In addition, asking each parent to complete their Journal on their own, and without discussion, exposed the different ways in which individuals interpreted this rather unconventional probe. As illustrated in our findings, this helped to reveal more about the individual practices and priorities of each parent.

In addition to capturing individual responses, our Family Tree probe also asked each set of parents to compare and negotiate their individual responses with each other, in order to create a collective response to the same task. This was intended to understand how parents might communicate and negotiate their individual perspectives within family life. As far as we are aware, this is the first time that a combination of individual and collective responses to the same probe have been used to explore the individual perspectives of family members. By comparing individual and collective Family Trees, we were able to discover aspects of family dynamics and relationships that would have been otherwise challenging to expose, had we relied solely on either individual or collective responses. As described in the findings, this process of asking sets of parents to first complete a task individually, and then to repeat it as a collective exercise, spurred interesting dialogues between them. The opportunities for collaborative dialogical sensemaking [29, 35] created by this task helped to raise parents awareness of each other's perceptions, and their own, which sometimes led to unexpected realisations that even surprised some of our participants. Though somewhat inadvertently, these that went on to play a pivotal role in surfacing subsequent discussions during Closing Interviews.

A probe approach to explore complex family experiences Reflections of our findings have led to a number of methodological insights. These insights pertain to the various ways in which to effectively use probes to tease out complex, tacit and even conflicting experiences that take place within families. Our approach to probes sought to find a balance between the individualistic and collective focus previous given to working with families. Our findings show that by taking this approach, our probes helped us to address some of the challenges posed by exploring family experiences of technology. Now we discuss these findings more broadly to provide those researchers, interested in exploring the individual perspectives on technology use that exist within families, with more general insights into how to approach the use of probes.

Capturing individual responses from multiple family members is required before we can compare them. Thus, allowing multiple family members to respond individually to probes is essential when attempting to explore their different individual perspectives on technology use and to enable more a complex and complete view of their experiences within everyday family life [23]. However, we acknowledge that this presents researchers with additional considerations. 
Firstly, this requires us to recruit multiple family members and to engage them in our probe activities. As discussed, family life is busy [34] and individual family members have different interests, needs and priorities [23]. Therefore, while researchers can intend to engage with all family members equally, it should be accepted that their individual levels of interest, effort, abilities and overall engagement may vary. This is heightened when including children's responses [23]. While this might limit the precision and confidence with which individual probe responses can be compared, the varying ways in which individual participants interpret probes can sometimes provide clues and stimulate interesting discussions about the individual perspectives of family members. Secondly, allowing multiple family members to respond individually to probes introduces two stages of dataanalysis; considering each participant's responses individually, and then within the responses of their family members. This adds complexity and time to this process.

While it is also essential to capture collective responses from multiple family members, relying solely upon their collective responses limits our ability to develop complete views on family experiences. This is because collective responses overlook the individual perspectives of individual family members and may instead amplify the views of more powerful, assertive or vocal individuals within the family [34]. Also, when attempting to capture collective responses, it is important to that some probes are better suited to capture collective responses than others. These are usually creative, fun, collaborative tasks that allow participants to express themselves within a relatively short and flexible timeframe. Given the shared, public nature of these tasks, collective responses will likely require more interpretation by researchers and offer limited depth. Therefore, to make these responses more useful, researchers might look for ways in which to offer participants a sense of privacy within these collective tasks. Probes designed to incorporate a sense of individual and collective duality might go some way to achieving this, as shown by our Family Experience Jar and Family Tree probes.

Including a probe that asks multiple family members to compare their individual responses to a task, and then to collaborate on a collective response significantly enhanced our approach. This is primarily because this process sparked discussions between family members, helping to raise their awareness of each other's perspectives, and of their own. These discussions also prepared participants for follow-up interviews in which we could more easily encourage and support participants to reflect on highly personal, sensitive and sometimes uncomfortable experiences of family technology use. Our approach also incorporated a combination of collective and individual interviews. Collective interviews are more suited to introduce probes. They ensure that individuals see themselves as part of a family unit and prompt them to reflect on experiences within family life. In contrast, individual interviews allow candid reflection on personal experiences of family life that might be considered embarrassing or socially undesirable [11]. While this aspect of our approach is beneficial, it introduces further time requirements both in conducting probe studies and analysing data.

As discussed, existing guidance on the use of probes e.g.[33, 53] tends to either consider an individual or collective approach [23]. Seeking a balance between these two approaches surfaced additional considerations, some of we have discussed. These considerations of how we can approach the use of probes to better understand family experiences of technology provide a significant contribution to researchers wishing to research co-experiences of technology use in families and other social groups.

\section{CONCLUSION}

Family experiences of technology use have been shown to be complex and messy. In particular, family conflict and tensions can arise when sets of parents have differing attitudes and approaches to technology use. This paper presents an example of how to effectively use probes to explore and compare the individual perspectives that exist within sets of parents. It describes the novel approach we took to using probes, by considering parents not just as being part of a set of parents, but also as individuals. It explains how we achieved this by designing our probe collection to capture a combination of individual and collective responses from each set of parents, and to stimulate discussions between them.

This novel approach to using probes helped to address some of the significant challenges posed by researching complex family experiences of technology. Firstly, developing our understanding of the social contexts in which these experiences take place. Secondly, raising our participants' awareness of each other's perspectives, as well as their own. Our approach allowed us to effectively use probes to tease out complex, tacit and even conflicting experiences that take place within families. This demonstration of how we can advance methods in HCI to help develop our understandings of the social experiences of technology use that increasingly permeate everyday life.

\section{LIMITATIONS AND RECOMMENDATIONS}

Our work has demonstrated the utility of using probes to collect a combination of individual and collective responses from multiple family members. We plan to extend this approach to include all family members e.g. children, and to explore a wider range of family configurations e.g. separated parents. This approach to using of probes could also consider how family boundaries and technology adoption evolve over time [43], for example, as children grow up.

Given the lack of explicit guidance on how to design probes to explore social experiences of technology, we see value in adapting this approach to develop more complete understandings of the perspectives of multiple people. We believe this a critical step in advancing methods to support the design of increasingly social interactive systems. 


\section{REFERENCES}

[1] Tawfiq Ammari, Priya Kumar, Cliff Lampe and Sarita Schoenebeck. 2015. Managing Children's Online Identities: How Parents Decide What to Disclose About Their Children Online. Proceedings of the 33rd Annual ACM Conference on Human Factors in Computing Systems. ACM, 1895-1904.

[2] Madeline Balaam, Judy Robertson, Geraldine Fitzpatrick, Rebecca Say, Gillian Hayes, Melissa Mazmanian and Belinda Parmar. 2013. Motherhood and HCI. CHI'13 Extended Abstracts on Human Factors in Computing Systems. ACM, 3215-3218.

[3] Katja Battarbee. 2003. Defining Co-Experience. Proceedings of the 2003 International Conference on Designing Pleasurable Products and Interfaces. ACM, 109-113.

[4] Lindsay Blackwell, Emma Gardiner and Sarita Schoenebeck. 2016. Managing Expectations: Technology Tensions among Parents and Teens. Proceedings of the 19th ACM Conference on Computer-Supported Cooperative Work \& Social Computing. ACM, San Francisco, California, USA, 1390-1401.

http://dx.doi.org/10.1145/2818048.2819928

[5] Kirsten Boehner, Janet Vertesi, Phoebe Sengers and Paul Dourish. 2007. How HCI Interprets the Probes. Proceedings of the SIGCHI Conference on Human Factors in Computing Systems. ACM, San Jose, California, USA, 1077-1086. http://dx.doi.org/10.1145/1240624.1240789

[6] Danah Boyd. 2014. It's Complicated: The Social Lives of Networked Teens. Yale University Press,

[7] Jennifer W Bryce and Hope Jensen Leichter. 1983. The Family and Television: Forms of Mediation. Journal of Family Issues 4, 2, 309-328.

[8] Thomas Dalsgaard, Mikael B. Skov, Malthe Stougaard and Bo Thomassen. 2006. Mediated Intimacy in Families: Understanding the Relation between Children and Parents. Proceedings of the 2006 Conference on Interaction Design and Children. ACM, Tampere, Finland, 145-152. http://dx.doi.org/10.1145/1139073.1139110

[9] Eleanor Chin Derix and Tuck Wah Leong. 2018. Days of Our Lives: Family Experiences of Digital Technology Use. Proceedings of the 30th Australian Conference on Computer-Human Interaction. ACM, Melbourne, Australia, 332-337. http://dx.doi.org/10.1145/3292147.3292185

[10] Eleanor Chin Derix and Tuck Wah Leong. 2019. Towards a Probe Design Framework. Proceedings of the 31st Australian Conference on Human-ComputerInteraction. 117-127.
[11] Audrey Desjardins, Ron Wakkary and William Odom. 2015. Investigating Genres and Perspectives in HCI Research on the Home. Proceedings of the 33rd Annual ACM Conference on Human Factors in Computing Systems. ACM, Seoul, Republic of Korea, 3073-3082.

http://dx.doi.org/10.1145/2702123.2702540

[12] Nicholas Epley, Adam Waytz and John T Cacioppo. 2007. On Seeing Human: A Three-Factor Theory of Anthropomorphism. Psychological Review 114, 4, 864.

[13] Jerry Fails, Mona Leigh Guha, Michael Horn and Sara Isola. 2012. Technology for Today's Family. CHI '12 Extended Abstracts on Human Factors in Computing Systems. ACM, Austin, Texas, USA, 2739-2742. http://dx.doi.org/10.1145/2212776.2212709

[14] Hasan Shahid Ferdous, Bernd Ploderer, Hilary Davis, Frank Vetere and Kenton O'Hara. 2015. Pairing Technology and Meals: A Contextual Enquiry in the Family Household. Proceedings of the Annual Meeting of the Australian Special Interest Group for Computer Human Interaction. ACM, Parkville, VIC, Australia, 370-379. http://dx.doi.org/10.1145/2838739.2838780

[15] Bill Gaver, Tony Dunne and Elena Pacenti. 1999. Design: Cultural Probes. Interactions 6, 1, 21-29. http://dx.doi.org/10.1145/291224.291235

[16] Lorna Gibson and Vicki L Hanson. 2013. Digital Motherhood: How Does Technology Help New Mothers? Proceedings of the SIGCHI Conference on Human Factors in Computing Systems. ACM, 313322.

[17] Victoria Haines, Val Mitchell, Catherine Cooper and Martin Maguire. 2007. Probing User Values in the Home Environment within a Technology Driven Smart Home Project. Personal Ubiquitous Comput. 11, 5, 349-359. http://dx.doi.org/10.1007/s00779-0060075-6

[18] R. Harper, T. Rodden, Y. Roggers and A. Sellen. 2008. Being Human: Hci in 2020. Microsoft, Cambridge, $U K$.

[19] Katherine M Hertlein. 2012. Digital Dwelling: Technology in Couple and Family Relationships. Family Relations 61, 3, 374-387.

[20] Alexis Hiniker, Sarita Y Schoenebeck and Julie A Kientz. 2016. Not at the Dinner Table: Parents' and Children's Perspectives on Family Technology Rules. Proceedings of the 19th ACM Conference on Computer-Supported Cooperative Work \& Social Computing. ACM, 1376-1389.

[21] Alexis Hiniker, Kiley Sobel, Hyewon Suh, Yi-Chen Sung, Charlotte P Lee and Julie A Kientz. 2015. 
Texting While Parenting: How Adults Use Mobile Phones While Caring for Children at the Playground. Proceedings of the 33rd annual ACM conference on human factors in computing systems. ACM, 727-736.

[22] Alexis Hiniker, Hyewon Suh, Sabina Cao and Julie A. Kientz. 2016. Screen Time Tantrums: How Families Manage Screen Media Experiences for Toddlers and Preschoolers. Proceedings of the 2016 CHI Conference on Human Factors in Computing Systems. ACM, San Jose, California, USA, 648-660. http://dx.doi.org/10.1145/2858036.2858278

[23] Willem Horst, Ties Bunt, Stephan Wensveen and Lisa Cherian. 2004. Designing Probes for Empathy with Families. Proceedings of the Conference on Dutch Directions in HCI. ACM, Amsterdam, The Netherlands, 15. http://dx.doi.org/10.1145/1005220.1005239

[24] Hilary Hutchinson, Wendy Mackay, Bo Westerlund, Benjamin B. Bederson, Allison Druin, Catherine Plaisant, Michel Beaudouin-Lafon, Helen Evans, Heiko Hansen and Nicolas Roussel. 2003. Technology Probes: Inspiring Design for and with Families. Proceedings of the SIGCHI Conference on Human Factors in Computing Systems. ACM, Ft. Lauderdale, Florida, USA, 17-24. http://dx.doi.org/10.1145/642611.642616

[25] Sara Isola and Jerry Alan Fails. 2012. Family and Design in the IDC and CHI Communities. Proceedings of the 11th International Conference on Interaction Design and Children. ACM, Bremen, Germany, 40-49. http://dx.doi.org/10.1145/2307096.2307102

[26] Heekyoung Jung, Erik Stolterman, Will Ryan, Tonya Thompson and Marty Siegel. 2008. Toward a Framework for Ecologies of Artifacts: How Are Digital Artifacts Interconnected within a Personal Life? Proceedings of the 5th Nordic conference on Human-Computer Interaction. 201-210.

[27] Nicholas Kardaras. 2016. Glow Kids: How Screen Addiction Is Hijacking Our Kids-and How to Break the Trance. St. Martin's Press,

[28] Priya Kumar and Sarita Schoenebeck. 2015. The Modern Day Baby Book: Enacting Good Mothering and Stewarding Privacy on Facebook. Proceedings of the 18th ACM Conference on Computer Supported Cooperative Work \& Social Computing. ACM, 13021312.

[29] Tuck Wah Leong, Peter Wright, Frank Vetere and Steve Howard. 2010. Understanding Experience Using Dialogical Methods: The Case of Serendipity. Proceedings of the 22nd Conference of the ComputerHuman Interaction Special Interest Group of
Australia on Computer-Human Interaction. ACM, 256-263.

[30] S. Livingstone and E. Helsper. 2007. New Media \& Society 9, 4, 671 .

[31] Sonia Livingstone and Ellen J. Helsper. 2008. Parental Mediation of Children's Internet Use. Journal of Broadcasting \& Electronic Media 52, 4, 581-599. http://dx.doi.org/10.1080/08838150802437396

[32] Clare Madge and Henrietta O'connor. 2006. Parenting Gone Wired: Empowerment of New Mothers on the Internet? Social \& Cultural Geography 7, 2, 199-220.

[33] Tuuli Mattelmäki. 2006. Design Probes. Aalto University,

[34] Melissa Mazmanian and Simone Lanette. 2017. Okay, One More Episode: An Ethnography of Parenting in the Digital Age. Proceedings of the 2017 ACM Conference on Computer Supported Cooperative Work and Social Computing. ACM, 2273-2286.

[35] John McCarthy and Peter Wright. 2004. Technology as Experience. Interactions 11, 5, 42-43.

[36] Brandon T. McDaniel, Adam M. Galovan, Jaclyn D. Cravens and Michelle Drouin. 2018. "Technoference" and Implications for Mothers' and Fathers' Couple and Coparenting Relationship Quality. Computers in Human Behavior 80, 303-313. http://dx.doi.org/https://doi.org/10.1016/j.chb.2017.11. 019

[37] Brandon T. McDaniel and Jenny S. Radesky. 2018. Technoference: Longitudinal Associations between Parent Technology Use, Parenting Stress, and Child Behavior Problems. Pediatric Research. http://dx.doi.org/10.1038/s41390-018-0052-6

[38] David Morley and Roger Silverstone. 1990. Domestic Communication-Technologies and Meanings. Media, culture \& society 12, 1, 31-55.

[39] Meredith Ringel Morris. 2014. Social Networking Site Use by Mothers of Young Children. Proceedings of the 17th ACM conference on Computer supported cooperative work \& social computing. ACM, 12721282.

[40] Carol Moser, Sarita Y Schoenebeck and Katharina Reinecke. 2016. Technology at the Table: Attitudes About Mobile Phone Use at Mealtimes. Proceedings of the 2016 CHI Conference on Human Factors in Computing Systems. ACM, 1881-1892.

[41] Carman Neustaedter, Kathryn Elliot and Saul Greenberg. 2006. Interpersonal Awareness in the Domestic Realm. Proceedings of the 18th Australia conference on Computer-Human Interaction. ACM, $15-22$. 
[42] Leysia Palen and Amanda Hughes. 2007. When Home Base Is Not a Place: Parents' Use of Mobile Telephones. Personal Ubiquitous Computing 11, 5, 339-348. http://dx.doi.org/10.1007/s00779-006-0078-3

[43] Sandra Petronio. 2002. Boundaries of Privacy: Dialectics of Disclosure. Suny Press,

[44] Catherine Plaisant, Allison Druin and Hilary Hutchinson. 2002. Technologies for Families. CHI 'O2 Extended Abstracts on Human Factors in Computing Systems. ACM, Minneapolis, Minnesota, USA, 938939. http://dx.doi.org/10.1145/506443.506669

[45] Jenny S. Radesky, Caroline J. Kistin, Barry Zuckerman, Katie Nitzberg, Jamie Gross, Margot Kaplan-Sanoff, Marilyn Augustyn and Michael Silverstein. 2014. Patterns of Mobile Device Use by Caregivers and Children During Meals in Fast Food Restaurants. Pediatrics 133, 4, e843-e849. http://dx.doi.org/10.1542/peds.2013-3703

[46] Diane J. Schiano, Christine Burg, Anthony Nalan Smith and Florencia Moore. 2016. Parenting Digital Youth: How Now? Proceedings of the 2016 CHI Conference Extended Abstracts on Human Factors in Computing Systems. ACM, San Jose, California, USA, 3181-3189.

http://dx.doi.org/10.1145/2851581.2892481

[47] Sue Shellenbarger. 1999. Work \& Family: Essays from the" Work \& Family" Column of the Wall Street Journal. Ballantine Books,

[48] Sherry Turkle. 2017. Alone Together: Why We Expect More from Technology and Less from Each Other. Hachette UK,

[49] Elizabeth A. Vandewater, Seoung-Eun Park, Xuan Huang and Ellen A. Wartella. 2005. "No-You Can't Watch That": Parental Rules and Young Children's Media Use. The American Behavioral Scientist 48, 5, 608-623.
[50] J. Mitchell Vaterlaus, Troy E. Beckert, Sarah Tulane and Clare V. Bird. 2014. "They Always Ask What I'm Doing and Who I'm Talking To": Parental Mediation of Adolescent Interactive Technology Use. Marriage \& Family Review 50, 8, 691-713. http://dx.doi.org/10.1080/01494929.2014.938795

[51] Frank Vetere, Martin R Gibbs, Jesper Kjeldskov, Steve Howard, Florian'Floyd' Mueller, Sonja Pedell, Karen Mecoles and Marcus Bunyan. 2005. Mediating Intimacy: Designing Technologies to Support StrongTie Relationships. Proceedings of the SIGCHI Conference on Human Factors in Computing Systems. ACM, 471-480.

[52] Amy Voida and Elizabeth D. Mynatt. 2005. Conveying User Values between Families and Designers. CHI '05 Extended Abstracts on Human Factors in Computing Systems. ACM, Portland, OR, USA, 2013-2016. http://dx.doi.org/10.1145/1056808.1057080

[53] Jayne Wallace, John McCarthy, Peter C. Wright and Patrick Olivier. 2013. Making Design Probes Work. Proceedings of the SIGCHI Conference on Human Factors in Computing Systems. ACM, Paris, France, 3441-3450. http://dx.doi.org/10.1145/2470654.2466473

[54] Anne Weibert and Kai Schubert. 2010. How the Social Structure of Intercultural Computer Clubs Fosters Interactive Storytelling. Proceedings of the 9th International Conference on Interaction Design and Children. ACM, Barcelona, Spain, 368-371. http://dx.doi.org/10.1145/1810543.1810616

[55] Sarita Yardi and Amy Bruckman. 2011. Social and Technical Challenges in Parenting Teens' Social Media Use. Proceedings of the SIGCHI Conference on Human Factors in Computing Systems. ACM, 3237-3246. 